LBL --30606

DE92 004094

\title{
Amorphous Ge Bipolar Blocking Contacts on Ge Detectors
}

\author{
P.N. Luke, C.P. Cork, N.W. Madden, C.S. Rossington and M.F. Wesela \\ Engineering Science Department \\ Engineering Division \\ Lawrence Berkeley Laboratory \\ University of California, Berkeley CA, 94720
}

October 1991

This work was supported by the Director, Office of Energy Research,

Office of Health and Environmental Research,

U.S. Department of Energy under Contract No. DE-AC03-76SF00098. 


\title{
Amorphous Ge Bipolar Blocking Contacts on Ge Detectors
}

\author{
P. N. Luke, C. P. Cork, N. W. Madden, C. S. Rossington and M. F. Wesela \\ Lawrence Berkeley Laboratory \\ University of California, Berkeley, CA 94720
}

\begin{abstract}
Electrical contacts formed using sputtered amorphous Ge (a-Ge) films on high-purity Ge crystals were found to exhibit good blocking behavior in both polarities with low leakage currents. The a-Ge contacts have thin dead layers associated with them and can be used in place of lithium-diffused, ionimplanted or Schottky barrier contacts on Ge radiation detectors. Multi-electrode detectors can be fatricated with very simple processing steps using these contacts.
\end{abstract}

\section{INTRODUCTION}

Semiconductor nuclear radiation detectors are usually operated in a full depletion mode and blocking contacts are required to maintain low leakage currents and high electric fields for charge collection. Blocking contacts on Ge detectors typically consist of n-type contacts formed by lithium diffusion and p-type contacts formed by boron ion implantation. Metal Schottky barriers can also be used as ptype contacts. When a detector is reverse biased, the lithium diffused contact acts as a hole blocking electrode and the boron implanted or surface barrier contact acts as an electron blocking electrode. These contacts are relatively simple to produce and have been in use since the early days of high-purity germanium detector development. However, there are drawbacks in using these contacts. First, lithiuin diffused contacts have dead layers on the order of several hundred microns thick which are undesirable in some applications. Although thin n-type contacts have been developed using phosphorus ion implantation, they require elaborate processing steps and cannot withstand high electric fields [1]; phosphorus implanted contacts are therefore only used in special applications, such as charged-particle transmission detectors. Second, the fabrication of monolithic multi-element detectors using conventional contacts can be difficult because of the need for many processing steps, and the formation of fine contact structures on lithium diffused contacts is not feasible because of their large diffusion depths $[2,3]$.

An alternative to the contacts discussed above are the amorphous semiconductor contacts. The first experimental study of electrical junctions between amorphous $\mathrm{Ge}(\mathrm{a}-\mathrm{Ge})$ and crystalline Ge was published in 1964 [4]. The use of a-Ge to fabricate blocking contacts on Si radiation detectors was first reported in 1971 [5]. These contacts showed good bipolar blocking behavior, i.e. they can block both electron and hole injection. Amorphous Ge blocking contacts on high-purity $\mathrm{Ge}$ detectors were investigated in 1977 but the devices showed large variations in leakage currents [6]. In those early studies, the a-Ge was deposited by vacuum evaporations. More recently, a-Si, produced by chemical vapor deposition, was successfully used to fabricate blocking contacts on $\mathrm{Si}$ to produce position-sensitive detectors [7].

In this paper, we report on the performance of high-purity $\mathrm{Ge}$ radiation detectors with a-Ge contacts fabricated using $R F$ sputtering techniques. Preliminary results show that sputtered a-Ge contacts can be used as blocking contacts on $\mathrm{Ge}$ radiation detector with potential advantages over conventional contacts.

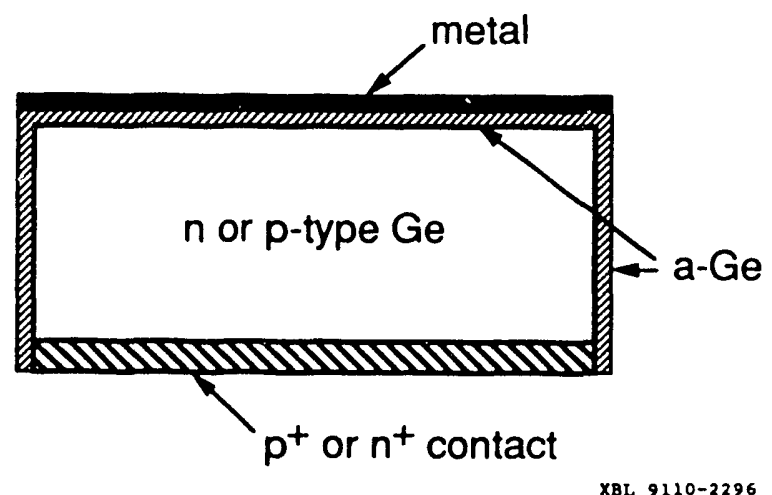

Fig. 1. Structure of the a-Ge contact.

\section{CONTACT FABRICATION}

Figure 1 shows the structure of an a-Ge contact on $\mathrm{Ge}$. For most of the devices reported here, conventional contacts (lithium diffused or boron ion implanted) were first formed on one side of the crystal before the fabrication of the a-Ge contact, although devices with a-Ge contacts on both sides were also fabricated. Prior to the a-Ge depositions, the $\mathrm{Ge}$ crystals were etched in a $4: 1 \mathrm{HNO}_{3}: \mathrm{HF}$ mixture, quenched in methanol and then blown dry with nitrogen. The a-Ge was deposited using an RF sputterer with a Ge target and a gas mixture of $7 \%$ hydrogen in argon. In fact, the sputtering parameters were identical to those used by our group in the deposition of a-Ge for $\mathrm{Ge}$ detector surface passivation [8]. Because of this, the a-Ge coating which was also deposited on the side of a device during formation of the a-Ge was left intact to function as a surface passivation layer. After the evaporation of the top metal layer which defines the a-Ge contact area, the device was tested without further processing of the side surface. The metal layer was deposited by vacuum evaporation from a filament. Gold was used as the metal layer for most of the contacts reported here but similar results were also obtained with contacts made using aluminium. Typical thicknesses for the a-Ge and the metal layers were $3000 \AA$ and $500 \AA$, respectively. 


\section{CONTACT EVALUATION}

Detectors with a-Ge contacts were fabricated using both $\mathrm{n}$ and p-type high-purity Ge crystals with net donor or acceptor concentrations of around $1 \times 10^{10} \mathrm{~cm}^{-3}$. Their I-V characteristics, spectroscopic and noise performance, window effects for low energy $x$ rays, and multi-electrode fabrication were evaluated. All the devices reported here had diameters of 33 to $38 \mathrm{~mm}$ and thicknesses of 8 to $11 \mathrm{~mm}$, except for those used in the $x$-ray measurements. In the following, we will use "a" to denote the a-Ge contact, e.g., a-p-p" represents a p-type detector with an $\mathrm{a}-\mathrm{Ge}$ contact on one side and a $\mathrm{p}^{+}$contact on the opposite side.

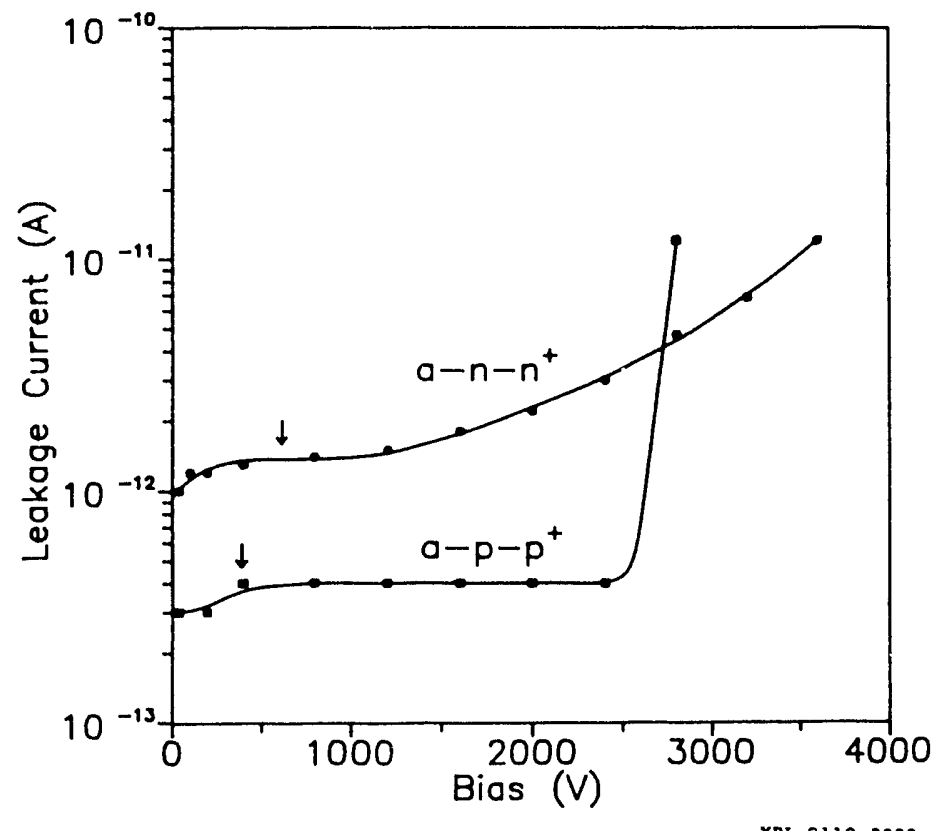

Fig. 2. Leakage current vs. reverse bias voltage of an a-n-n ${ }^{+}$ and an a-p-p ${ }^{+}$detector at a temperature of $79 \mathrm{~K}$. The arrows indicate the full depletion voltages.

\section{I-V Characteristics}

I-V measurements were performed using a liquid nitrogen cooled cryostat with the detector temperature held nominally at $79 \mathrm{~K}$. Variable temperature leakage current measurements were made by removing the liquid nitrogen from the cryostat and allowing the detector temperature to drift slowly upward. Inside the cryostat, a cooled shield surrounded the detector to prevent room temperature IR radiation from reaching the detector and producing additional leakage currents. Leakage currents were measured using an electrometer. Figure 2 shows reverse I-V characteristics of an $a-n-n^{+}$device and an a-p-p $p^{+}$device. Full depletion voltages are indicated by the arrows. In both cases, depletion starts from the a-Ge contacts and therefore electric fields existed at the a-Ge contacts even at below full depletion bias voltages. The a-Ge contacts displayed excellent blocking behavior with very low leakage currents even under high clectric fields $(>3000 \mathrm{~V} / \mathrm{cm})$. The side surfaces of the devices and the opposite contacts could also have contributed to the total leakage currents measured.

Figure 3 shows the temperature dependence of the leakage current for an a-p- $\mathrm{p}^{+}$and an $\mathrm{a}-\mathrm{n}-\mathrm{n}^{+}$device. For comparison, the leakage currents of the same devices with conventional contacts are also shown. The $a-n-n^{+}$device shows a much higher leakage current than the a-p- $\mathrm{p}^{+}$and conventional devices above liquid nitrogen temperatures.

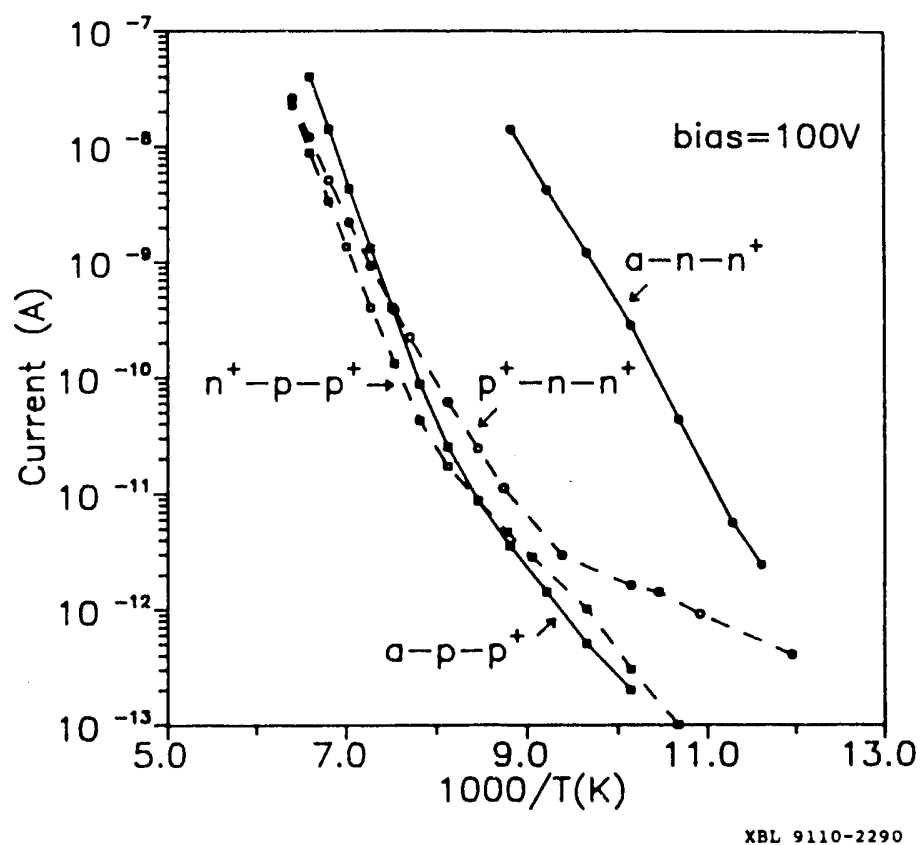

Fig. 3. Reverse leakage current vs. temperature of an $a-n-n^{+}$ and an a-p- $\mathrm{p}^{+}$detector compared to the same detectors with conventional contacts.

Since the a-Ge contacts are non-injecting in both polarities, they can replace both the $\mathrm{n}^{+}$and the $\mathrm{p}^{+}$contacts of a detector. Figure 4a shows capacitance as a function of voltage for an a-p-a device. As with a conventional $\mathrm{p}$-i-n device, the depletion width increased and the capacitance decreased with increasing voltage until full depletion was reached. The device was operated at voltages above full depletion with the back, negatively biased, contact acting as an electron blocking electrode. However, when the voltage was returned to zero, the capacitance remained constant because there was no carricr injection from the back contact which was then acting as a hole blocking electrode; the device remained fully depleted. At this point, both contacts were at the same potential and a potential minimum for holes existed at the mid-plane of the device due to the presence of space charge from the ionized impurities. The capacitance gradually increased with time because holes generated by background radiations and leakage current collected at the potential minimum and built up an undepleted layer of increasing thickness at the middle of the device. Irradiation with a gamma ray source can spced up this process as shown in Fig. $4 \mathrm{~b}$. 

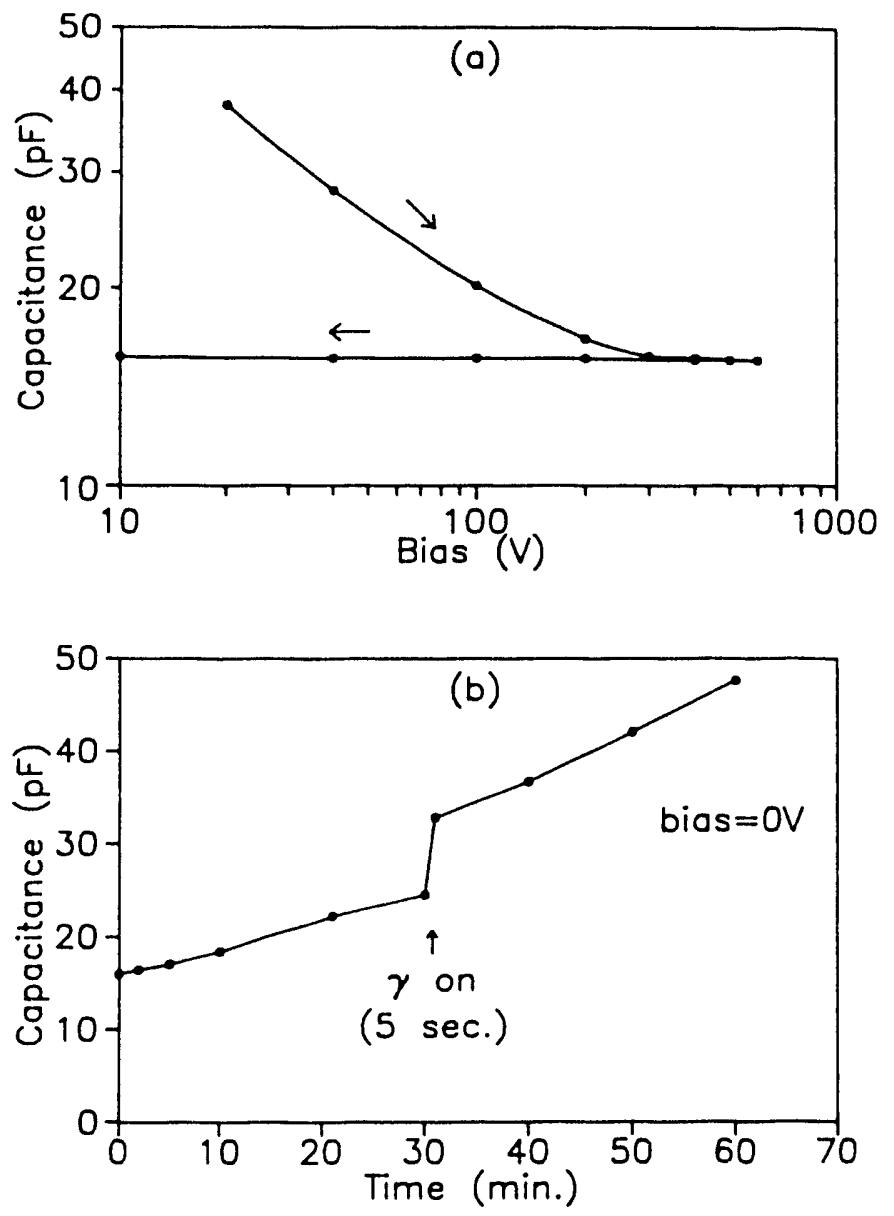

XBL $9110-2291$

Fig. 4. (a) C-V characteristics of an a-p-a detector and (b) the slow recovery from full depletion because of the non-injecting contacts.

\section{Spectroscopic Performance}

Detectors with a-Ge contacts performed spectroscopically as well as those with conventional contacts. $A{ }^{60} \mathrm{Co}$ spectrum taken using an $a-n-n^{+}$detector is shown in Fig. 5. No undesirable effects associated with the a-Ge contacts were observed.

Noise measurements were obtained for an a-p- $\mathrm{p}^{+}$detector with a pulsed-opto reset charge-sensitive amplifier employing a cooled FET (2N6453) input stage. Another set of measurements were made after the a-Ge contact was replaced with a lithium diffused contact. In both cases, the side of the detector was coated with a-Ge. The results are shown in Fig. 6. The series noise, $1 / f$ noise and parallel noise components were obtained by fitting to the measured noise levels. The series noise component was consistent with the expected FET noise in conjunction with a detector capacitance of $20 \mathrm{pF}$, i.e. there were no significant series noise contributions from either the a-Ge or the lithium diffused contacts. The difference in the parallel noise levels is accounted for by the different leakage currents observed between the two devices. It is difficult to draw conclusions regarding the $1 / \mathrm{f}$ noise component since it was apparently dominated by the electronic system. Additional measurements using improved electronics are needed to determine if there are significant $1 / f$ noise contributions from the a-Ge contacts.

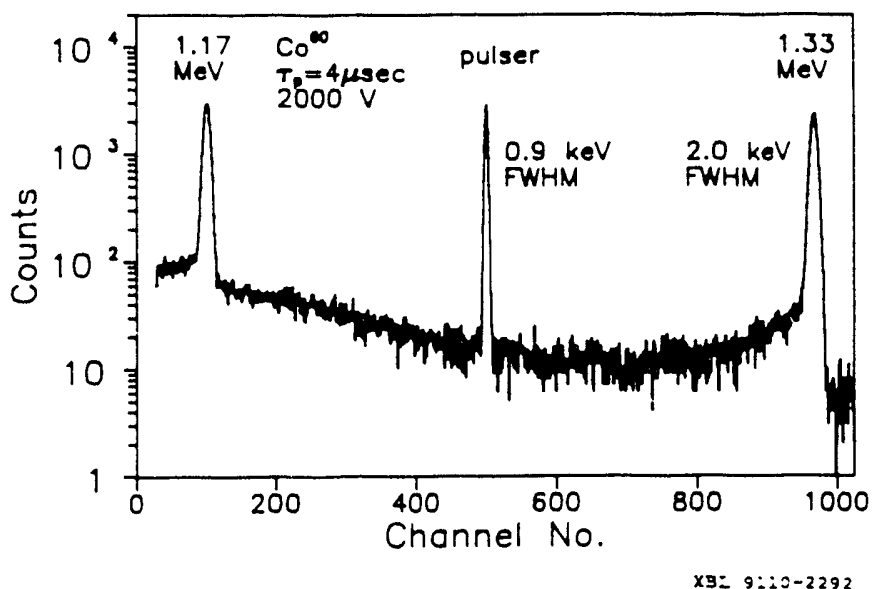

Fig. 5. A ${ }^{60}$ Co spectrum taken using an a-p- $\mathrm{p}^{+}$detector.

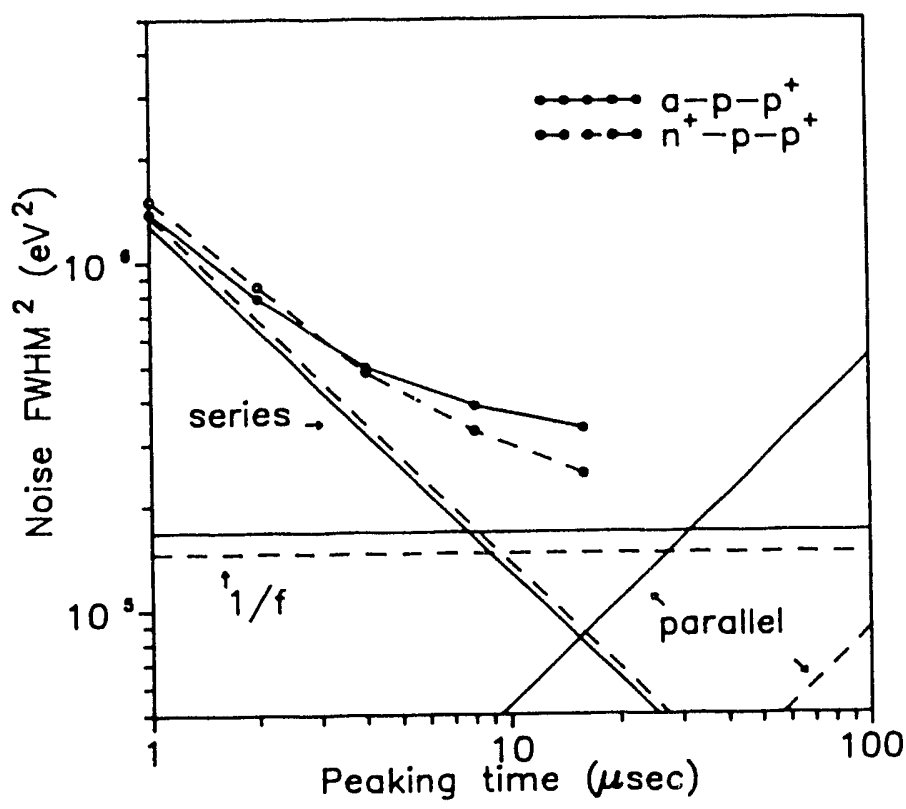

XBL $9110-2293$

Fig. 6. Electronic noise vs. peaking time measured with a pulsed-opto reset charge-sensitive amplifier connected to an a$\mathrm{p}-\mathrm{p}^{+}$detector and an $\mathrm{n}^{+}-\mathrm{p}-\mathrm{p}^{+}$detector.

\section{Window Effects}

The a-Ge contacts are much thinner than lithium diffused contacts and therefore have good transmission extending to the low energy $x$-ray range. However, at sufficiently low energies, where photon absorption occurs very close to the entrance contact, window effects arising from incomplete charge collection become important. Such effects give rise to spectral 
background below the full energy peaks and have been observed in Ge detectors with Schottky barrier [9] and ion implanted $[10,11]$ contacts. The magnitude of the window effect is generally larger in Ge than in Si because of the higher absorption coefficient. This has inhibited the use of Ge detectors in low energy $x$-ray spectroscopy applications. Ge detectors with $\mathrm{Ni}$ evaporated contacts have been reported to have very small window effects [12], but we were unable to reproduce such results in our laboratory.

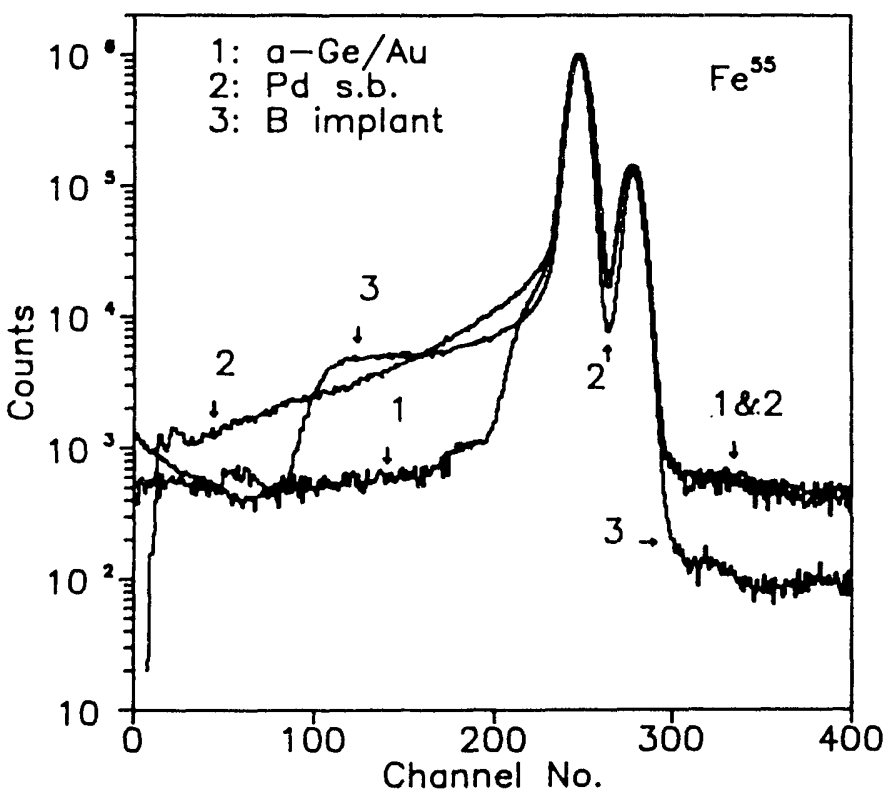

XBL $9110-2294$

Fig. 7. ${ }^{55} \mathrm{Fe}$ spectra obtained from Ge detectors with (1) a$\mathrm{Ge} / \mathrm{Au},(2) \mathrm{Pd}(500 \AA)$ Schottky barrier and (3) boron ion implanted $\left(25 \mathrm{keV}, 1 \times 10^{14} \mathrm{~cm}^{-2}\right)$ entrance window contacts.

The low energy spectral response of an a-p- $n^{+}$detector was measured using $\mathrm{Mn} \mathrm{K} \times$-rays entering through the a-Ge contact. As a comparison, Fig. 7 shows ${ }^{55} \mathrm{Fe}$ spectra taken using Ge detectors with an a-Ge contact, a Pd Schottky barrier contact and a boron ion implanted contact as the entrance window. All three detectors have "top-hat" structures, each with an active area of $20 \mathrm{~mm}^{2}$ and thickness of $5 \mathrm{~mm}$. The spectrum obtained using the detector with the a-Ge contact showed much lower background below the main photo-peak compared to the other detectors. The a-Ge contact of this detector consisted of a $3000 \AA$ thick layer of a-Ge and a $300 \AA$ thick layer of $\mathrm{Au}$. We are investigating the factors affecting the spectral background and hope to further reduce or eliminate the "shoulder" that is present on the low energy side of the main photopeak.

\section{Multi-electrode Detectors}

One of the most attractive features of the a-Ge contact is the ease with which multi-electrode detectors can be fabricated.
Because the a-Ge coating used for contact formation is the same as that used for surface passivation, both can be accomplished in one processing step, eliminating the need for additional surface treatments after the contacts are formed. As illustrated in Fig. 8, multiple electrodes can be formed on a device simply by sputtering with a-Ge followed by metal evaporation, e.g. through a shadow mask, to define the contact areas. Areas between the electrodes are automatically passivated. It may also be possible to fabricate double-sided multi-electrode detectors using this technique.

For test purposes, a two segment a-p- $\mathrm{p}^{+}$detector has been fabricated by performing the metal evaporation through a line mask. The resulting gap between the two electrodes was 0.5 $\mathrm{mm}$ wide and $3 \mathrm{~cm}$ long. Figure 9 shows the leakage currents of the two individual segments as a function of bias voltage. The interelectrode resistance was measured by monitoring the leakage current of one segment while varying the potential of the other segment. No detectable change in current $\left(<1 \times 10^{-13}\right.$ A) was observed with a voltage variation of $\pm 10 \mathrm{~V}$, which implies that the interelectrode resistance was $>1 \times 10^{14}$ ohms.

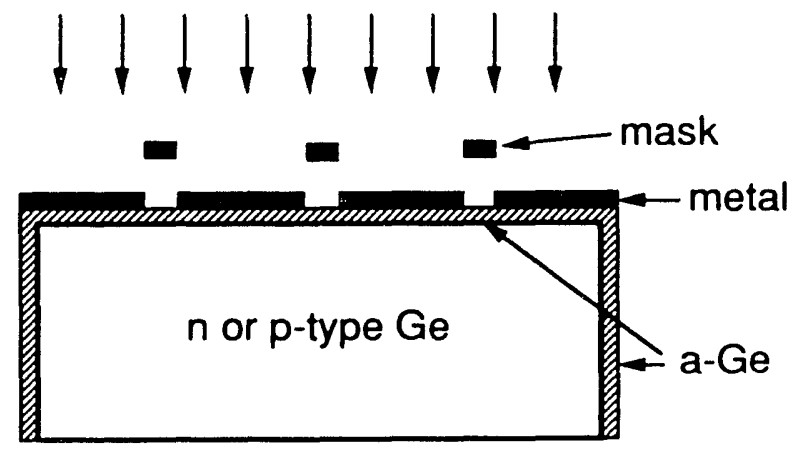

XBL $9110-2297$

Fig. 8. Multi-electrode detector construction using a-Ge contacts.

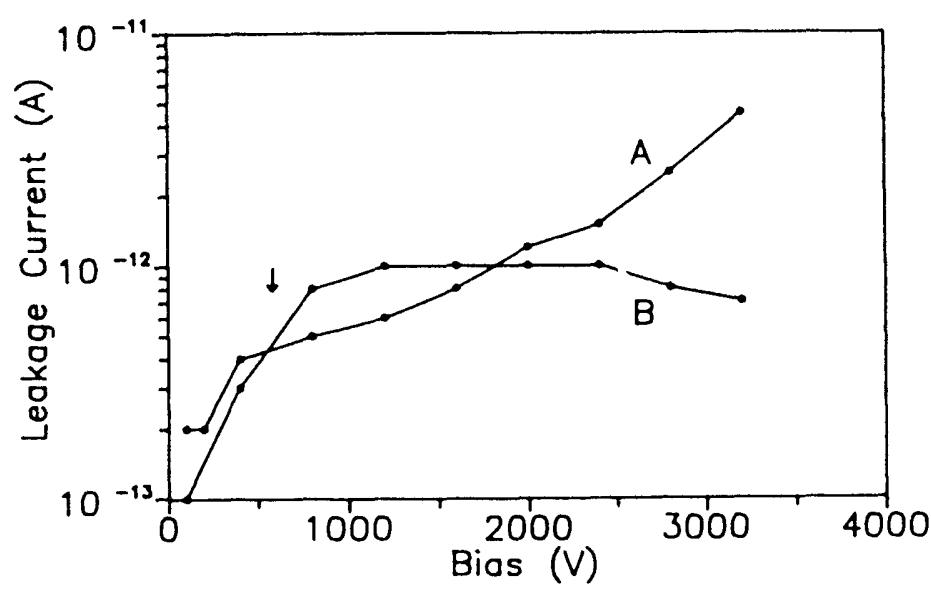

XBL $9110-2295$

Fig. 9. Leakage current from each of the two segments of an a$\mathrm{p}-\mathrm{p}^{+}$detector. 


\section{CONCLUSIONS}

Preliminary tests have shown that a-Ge contacts on Ge detectors exhibit excellent bipolar blocking properties and that they possess advantageous features compared to conventional contacts. However, further studies need to be carried out to understand how the contacts function, to determine what factors affect their performance, and to fully characterize them. For example, one important issue which has not yet been investigated is the stability of the a-Ge contact with heat treatments, since, in practice, detectors often need to be heated either during vacuum bake-outs in cryostats or for the repair of radiation damage.

\section{ACKNOWLEDGMENT}

We wish to thank Bradley Krieger for his assistance in fabricating some of the detectors. This work was supported by the Director's Office of Energy Research, Office of Health and Environmental Research, of the U.S. Department of Energy under Contract No. DE-AC03-76SF00098.

\section{REFERENCES}

[1] R.H. Pehl, P.N. Luke and D.L. Friesel, "High-purity Germanium Charged-particle Detectors: A LBL-IUCF Update," Nucl. Instr. and Meth., vol. A242, pp. 103-110, Dec. 1985.

[2] D. Gutknecht, "Photomask Technique for Fabricating High Purity Germanium Strip Detectors," Nucl. Instr. and Meth. vol. A288, pp. 13-18, Mar. 1990.

[3] P.N. Luke, "Gold-mask Technique for Fabricating Segmentedelectrode Germanium Detectors," IEEE Trans. Nucl. Sci., vol. NS-31, pp. 312-315, Feb. 1984.

[4] R. Grigorovici, N. Croitoru, A. Devenyi and E. Teleman, "Band Structure and Electrical Conductivity in Amorphous Germanium," Proc. 7th International Conference on the Physics of Semiconductors, Paris, France, July 1964, pp. 423-428.

[5] J.B.A. England and V.W. Hammer, "A New Type of Noninjecting Back Contact for Totally Depleted Silicon Surface Barrier Detectors," Nucl. Instr. and Meth., vol. 96, pp. 8183, Sept. 1971.

[6] W.L. Hansen and E.E. Haller, "Amorphous Germanium as an Electron or Hole Blocking Contact on High-purity Germanium Detectors," IEEE Trans. Nucl. Sci., vol. NS-24, pp. 61-63, Feb. 1977

[7] Y. Chiba et al., "A Position-sensitive Radiation Detector Using a Heterojunction of Amorphous and Crystalline Silicon," Nucl. Instr. and Meth., vol. A299, pp. 152-156, Dec. 1990.

[8] W.L. Hansen, E.E. Haller and G.S. Hubbard, "Protective Surface Coatings on Semiconductor Nuclear Radiation Detectors," IEEE Trans. Nucl. Sci., vol. NS-27, pp. 247-251, Feb. 1980

[9] J. Llacer, E.E. Haller and R.C. Cordi, "Entrance Windows in Germanium Low-energy X-ray Detectors," IEEE Trans. Nucl. Sci., vol. NS-24, pp. 53-60, Feb. 1977.

[10] R.E. McMurray Jr., P.N. Luke, J.M. Jaklevic and J.T. Walton. "Effects of Germanium Detector Contacts on Dead Layer Performance for Soft X-rays," Stanford Synchrotron Radiation Workshop, October 1987.
[11] M. Slapa et al., "Preliminary Study of the Behavior of HPGe Detectors with Ion Implanted Contacts in the Ultralow-energy X-ray Region," Advances in X-ray Analysis, vol. 25, pp. 23 30, 1982.

[12] C.E. Cox, B.G. Lowe and R.A. Sareen, "Small Area High Purity Germanium Detectors for use in the Energy Range $100 \mathrm{eV}$ to $100 \mathrm{keV}, "$ IEEE Trans. Nucl. Sci., vol. NS-35, pp. 28-32, Feb. 1988. 

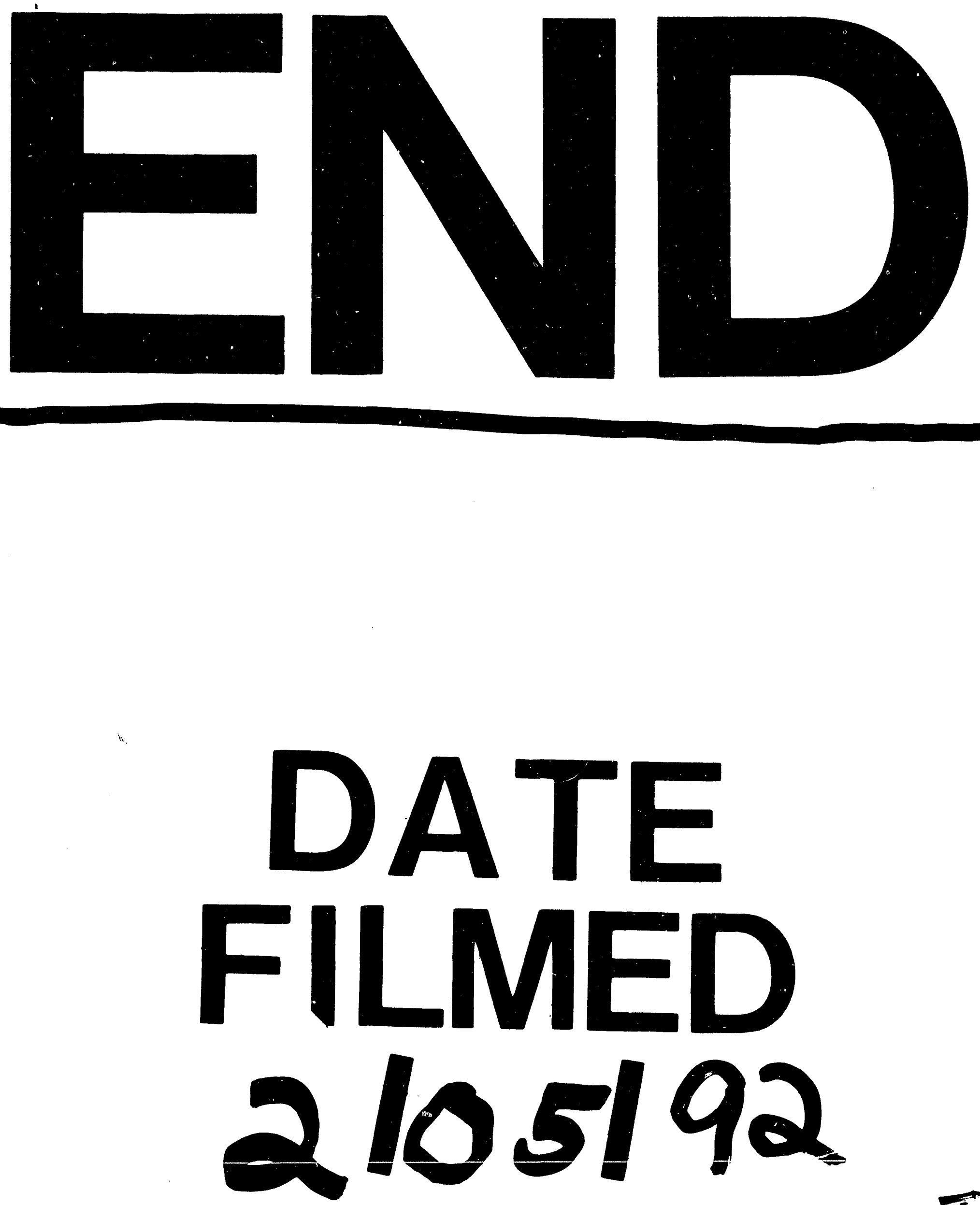

$I$ 


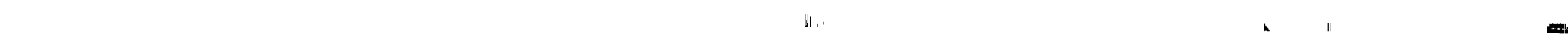

